\title{
Rogue waves under influence of Raman delay
}

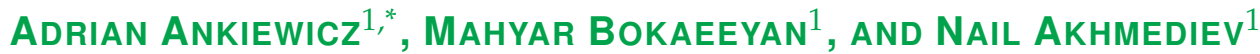 \\ ${ }^{1}$ Optical Sciences Group, Research School of Physical Sciences and Engineering, The Australian National University, Canberra ACT 2601, Australia \\ *Corresponding author: Adrian.Ankiewicz@anu.edu.au
}

Compiled February 23, 2018

\begin{abstract}
Rogue waves can appear in optical fibers and other optical systems, as well as in natural events like water waves. Their mathematical description is based on partial differential equations that have solutions that are localized both in time and space. One example is the Peregrine solution of the nonlinear Schrödinger equation (NLSE). When higher-order terms in the equation are involved, the solution becomes distorted, but its main features remain localized in space and time. Although exact solutions are not obtained in all cases, approximations which describe the solutions with reasonable accuracy do exist. Here, we consider approximate rogue wave solutions of the NLSE with an optically-relevant Raman delay term.
\end{abstract}

(C) 2018 Optical Society of America

OCIS codes: (060.5530) Pulse propagation and temporal solitons; (140.7090) Ultrafast lasers;(190.5650) Raman effect; (320.7110) Ultrafast nonlinear optics.

http://dx.doi.org/10.1364/ao.XX.XXXXXX

\section{INTRODUCTION}

Rogue waves were first found in optics as high-amplitude pulses in supercontinuum generation [1]. However, these are not the only type of rogue waves in optics. They can also be found in the output of laser radiation [2-6] and in other types of optical cavities [7-10]. They can be influenced by Brillouin scattering [11] or by the Raman effect [12]. Indeed, there is a multiplicity of forms of rogue waves [13-17], and this can make it difficult to classify them at present. Mathematically, the simplest type can be represented in the form of the 'Peregrine' solution of the nonlinear Schrödinger equation [18]. This is a solution that is localized both in time and in space. This fundamental solution can be extended to cover more complicated cases [19-22]. Rogue waves involving partial nonlinearity have been studied [23], and effects in media with decreasing dispersion or diffraction have been considered in [24]. Akhmediev breathers and rogue waves can also appear in PT -symmetric coupled waveguides [25]. However, presenting rogue wave solutions in exact form is not always possible.

Light propagation in optical fibers is subject to various higherorder effects [31], [32], e.g. third-order dispersion, $\psi_{t t t}$, fourthorder dispersion, $\psi_{t t t t}$, a 'quintic' adjustment to the Kerr nonlinearity, $|\psi|^{4} \psi$ and the Raman downshift effect, $\tau_{R} \psi\left(|\psi|^{2}\right)_{t}$. Similarly, water wave propagation in 'deep' water can be influenced by assorted physical phenomena [33], e.g. wind, viscosity and bottom friction, introducing terms like $\psi_{t t t},|\psi|^{2} \psi_{t}$ and $|\psi|^{1-\sigma} \psi$. These higher-order effects can potentially influence the dynamics of rogue waves [34] in ways that differ from their effect on solitons. Such impacts can significantly change the detected shapes. In this work, we consider the effects of Raman delay and other disturbances on the shape of rogue waves, and, where possible, compare the results with predictions from other possible approaches.

\section{BASIC EQUATIONS}

We start with the extended nonlinear Schrödinger equation that describes propagation of ultra-short pulses in optical fibres:

$$
i \psi_{x}+\frac{1}{2} \psi_{t t}+|\psi|^{2} \psi=R
$$

where $R$ indicates the additional terms in the form

$$
\begin{aligned}
R= & \tau_{R} \psi\left(|\psi|^{2}\right)_{t}-v|\psi|^{4} \psi-v_{2 n}|\psi|^{2 n} \psi \\
& +i \gamma_{3} \psi_{t t t}-d_{4} \psi_{t t t t}-d_{6} \psi_{t t t t t t}-i s\left(\psi|\psi|^{2}\right)_{t},
\end{aligned}
$$

where $n$ is an integer, $n=3,4, \cdots \infty$. Here $v$ represents a nonlinearity which is of higher order than a Kerr-law response for the system. To start with, we restrict ourselves to these examples of higher-order terms that are commonly studied in the literature. Other types of terms can be considered using the same technique as we discuss here.

Equation (1) in general is not integrable and cannot be solved exactly. Therefore, we have a choice of finding approximate solutions. Among the techniques that allows us to do that we can list the Lagrangian approach and the "method of moments". The Lagrangian technique was introduced into soliton theory 
by Anderson [26]. It can be used to understand the effects of various disturbances on a known system. The 'method of moments' [27] can also be used for reducing the dimensionality of soliton dynamical systems. The Lagrangian approach is based on minimization of "action". It can give results which are similar to those obtained by the 'method of moments' [28]. The two methods were compared in regard to the soliton solutions of the cubic-quintic complex Ginzburg - Landau equation in [29]. It has also been used to study self-focusing and self-defocusing two-dimensional beams in dissipative media [30].

It is less obvious that the Lagrangian approach can be applied to rogue wave solutions which are localized both in space and time, in contrast to soliton solutions that are localized only in either time or space. In this work, we show that the technique can be applied to rogue waves when the exact solutions may not be available. In particular, it can be applied to non-integrable equations such as higher-order extensions of the NLSE.

The use of the variational integral and Euler equations has been explained in [35], where the Lagrange density is introduced and its relation to the Euler-Lagrange equations for the Schrödinger equation and other conservative equations is also given. We employ the Lagrangian

$$
L=\int_{-\infty}^{\infty} L_{d} d t
$$

where $L_{d}$ is the Lagrangian density and we have

$$
L_{d}=-\frac{i}{2}\left(\psi^{*} \frac{\partial \psi}{\partial x}-\psi \frac{\partial \psi^{*}}{\partial x}\right)+\frac{1}{2}\left|\frac{\partial \psi}{\partial t}\right|^{2}-\frac{1}{2}|\psi|^{4}
$$

for field $\psi(x, t)$. When dealing with solutions on a constant background, it is common practice to subtract off any constant part of $L_{d}$.

If we apply the Euler-Lagrange equations to the above equation, we obtain:

$$
-\frac{\partial L_{d}}{\partial \psi^{*}}+\frac{\partial}{\partial x} \frac{\partial L_{d}}{\partial \psi_{x}^{*}}+\frac{\partial}{\partial t} \frac{\partial L_{d}}{\partial \psi_{t}^{*}}-\frac{\partial^{2}}{\partial t^{2}}\left(\frac{\partial L_{d}}{\partial \psi_{t t}^{*}}\right)=R,
$$

where a subscript $x$ or $t$ means derivative with respect to that variable, the star * indicates complex conjugate and $\mathrm{R}$ indicates the added higher-order terms.

For a trial solution containing several parameters $c^{(j)}, j=$ $1,2 \ldots . .$, the standard variational approach can be modified to allow for dissipative terms [28, 29, 36, 37]. In this way, we obtain a separate equation

$$
\begin{aligned}
& \frac{d}{d x}\left(\frac{\partial L}{\partial c_{x}^{j}}\right)-\frac{\partial L}{\partial c^{(j)}} \\
& =2 \operatorname{Re}\left(\int_{-\infty}^{\infty} R \frac{\partial \psi^{*}}{\partial c^{(j)}} d t\right) \equiv J\left[c^{j} ; R\right]
\end{aligned}
$$

for each parameter $c^{(j)}$. The set of Eqs.(4) comprise the reduced dynamical system for the rogue wave parameters. In other words, the wave profiles and, more generally, the wave evolution are described by the variation of these parameters.

\section{SIMPLE EXAMPLES}

\section{A. Peregrine rogue wave}

In order to illustrate the application of the above technique to solutions which are localized both in time and space, we first apply it to the simplest case, viz. the lowest-order rational solution of the NLSE. Thus, we first consider the case $R=0$, i. e. the basic nonlinear Schrödinger equation,

$$
i \psi_{x}+\frac{1}{2} \psi_{t t}+|\psi|^{2} \psi=0,
$$

and illustrate the idea using its known exact solution. Namely, we write the solution in the form of trial function

$$
\psi=\left[4 \frac{1+2 i x}{c(x)+4 t^{2}}-1\right] \exp [i x]
$$

with the intention of finding the unknown function, $c(x)$.

Following Eq.(3), we first write the Lagrangian,

$$
\begin{gathered}
L=-\frac{2 \pi}{c(x)^{7 / 2}}\left[-x c(x)^{2} c^{\prime}(x)-14\left(4 x^{2}+1\right) c(x)\right. \\
\left.+2 c(x)^{3}+10\left(4 x^{2}+1\right)^{2}\right] .
\end{gathered}
$$

Eq.(4) allows us to write the equation relative to the unknown function $c(x)$ :

$$
-\frac{70 \pi\left(4 x^{2}+1\right)\left(-c(x)+4 x^{2}+1\right)}{c(x)^{9 / 2}}=0,
$$

which results in

$$
c(x)=1+4 x^{2}
$$

giving

$$
\psi=\left[4 \frac{1+2 i x}{1+4 x^{2}+4 t^{2}}-1\right] \exp [i x]
$$

Clearly, this is the well-known rogue wave solution of the NLSE [19]. In this case, the result is exact, as we deal with the integrable NLSE and we knew the form of the solution in advance. In general, the solutions are expected to be approximate.

\section{B. 'Moving' rogue wave}

As a more complicated example, we consider a rogue wave with an internal 'velocity' or 'skewness'. From previous experience, we know that this occurs for rogue waves of the Hirota equation [38] and for a multiplicity of extended equations with odd higher-order terms [39]. In order to describe this type of rogue waves, we take the solution in the form

$$
\psi(x, t)=\left[4 \frac{1+2 i x}{D_{m}(x, t)}-1\right] e^{i F_{m}(x, t)}
$$

where

$$
D_{m}(x, t)=r(x)+4\left[t-t_{0}(x)\right]^{2}
$$

and

$$
F_{m}(x, t)=x+a(x)\left[t-t_{0}(x)\right]-\theta(x)
$$

with the function $t_{0}(x)$ being responsible for the lateral motion.

We now need to find $L$ and then the 4 unknown functions $a(x), t_{0}(x), r(x), \theta(x)$. We find:

$$
\begin{gathered}
L \frac{r^{7 / 2}(x)}{2 \pi}=r^{2}(x)\left[-2 X a(x) t_{0}^{\prime}(x)+X a^{2}(x)+x r^{\prime}(x)\right. \\
\left.-2 X \theta^{\prime}(x)\right]-r^{3}(x)\left[2-2 a(x) t_{0}^{\prime}(x)+a^{2}(x)-2 \theta^{\prime}(x)\right] \\
+14 X r(x)-10 X^{2}
\end{gathered}
$$

where, for convenience, we define $X=1+4 x^{2}$. 
We obtain the 4 ODEs, and then solve the $a(x)$ equation by setting $a(x)=t_{0}^{\prime}(x)$. The first (i.e. $\theta$ ) equation found is:

$$
r(x)\left[r^{\prime}(x)+16 x\right]-3\left(4 x^{2}+1\right) r^{\prime}(x)=0,
$$

and we use its simplest solution, viz.. $r(x)=4 x^{2}+1$.

Then the only remaining non-zero equation is:

$$
\left[t_{0}^{\prime}(x)\right]^{2}+2 \theta^{\prime}(x)=0
$$

Now $t_{0}^{\prime}(x)$ is a velocity, and the simplest solution is to take it as an arbitrary constant velocity, $\mathrm{v}$, so $t_{0}^{\prime}(x)=v$. Hence $t_{0}(x)=v x$ and $\theta(x)=-v^{2} x / 2$, so we have

$$
\psi=\left[4 \frac{1+2 i x}{1+4 x^{2}+4(t-v x)^{2}}-1\right] e^{i\left[v t+\left(1-\frac{v^{2}}{2}\right) x\right]} .
$$

The Galilean transformation of the NLSE is well-known (e.g. see Eq.(2.6) of [40]). The solution given by Eq.(11) agrees with that found by applying such a transform to the basic rogue wave of Eq.(6) in Sect.A. It reduces to that lowest-order rogue wave when $v=0$. We can see that the Lagrangian approach works well for these simple illustrative cases. This encourages us to move up to more complicated cases, as given below.

\section{MORE GENERAL FORM OF THE TRIAL FUNCTION}

As a next step, we allow for nonzero functional $\mathrm{R}$ in the trial function. One of the consequences may be resizing of the rogue wave, say, in the $x$-direction. In order to take this into account, we extend the result of Eq.(10), to allow for a stretching factor, B, by taking

$$
\psi(x, t)=\left[4 \frac{1+2 i B x}{D_{m}(x, t)}-1\right] e^{i F_{m}(x, t)},
$$

with $D_{m}(x, t)$ still given by Eq.(8) and $F_{m}(x, t)$ given by Eq.(9). Now, we apply the Euler-Lagrange formalism to the more general trial function (12).

For convenience, we split $L_{d}$ into three parts:

$$
L_{d}=L_{d 1}+L_{d 2}+L_{d 3}
$$

where

$$
\begin{gathered}
L_{d 1}=-\frac{i}{2}\left(\psi^{*} \frac{\partial \psi}{\partial x}-\psi \frac{\partial \psi^{*}}{\partial x}\right)+a_{1} a(x) t_{0}^{\prime}(x)+h_{1} \theta^{\prime}(x)-u_{1}, \\
L_{d 2}=\frac{1}{2}\left|\frac{\partial \psi}{\partial t}\right|^{2}
\end{gathered}
$$

and

$$
L_{d 3}=-\frac{1}{2}\left(|\psi|^{4}-1\right)
$$

We need to integrate these terms over an infinite range in $y_{1} \equiv t-t_{0}(x)$. The terms in odd powers of $y_{1}$ will integrate out to zero. In the remaining part of $L_{d 1}$, a part is subtracted so that its limit is zero for high $\left|y_{1}\right|$. Then, taking $L_{j}=\int_{-\infty}^{\infty} L_{d j} d t$, we have $L=L_{1}+L_{2}+L_{3}$.

Taking the limit $\lim _{y_{1} \rightarrow \infty} L_{d 1}$ shows that it equals to

$$
\left(a_{1}-1\right) a(x) t_{0}^{\prime}(x)+\left(h_{1}-1\right) \theta^{\prime}(x)-u_{1}+1,
$$

with unknown functions $a(x), t_{0}^{\prime}(x), \theta^{\prime}(x)$. Setting this to zero shows that the constants needed here are $a_{1}=h_{1}=u_{1}=1$. Now, $L_{d 2}$ clearly approaches zero for large $t$ or $y_{1}$, and so needs no level adjustment. For $L_{d 3}$, we note that $\lim _{y_{1} \rightarrow \infty}|\psi|=1$, hence we subtract this background before integrating. We thence find:

$$
\begin{aligned}
& L \frac{r^{7 / 2}(x)}{2 \pi}=14 X r(x)-10 X^{2}+r(x)^{2}\left[a^{2}(x) X\right. \\
& \left.-2 a(x) X t_{0}^{\prime}(x)-2 X \theta^{\prime}(x)+B x r^{\prime}(x)+4 B-4\right] \\
& -r(x)^{3}\left[a^{2}(x)+2 B-2 a(x) t_{0}^{\prime}(x)-2 \theta^{\prime}(x)\right]
\end{aligned}
$$

where $X=4 B^{2} x^{2}+1$.

Using Eq.(4), we define the left-hand-side terms of the equation relating to parameter $c^{(j)}$ as:

$$
S\left[c^{(j)}\right] \equiv \frac{d}{d x}\left(\frac{\partial L}{\partial c_{x}^{j}}\right)-\frac{\partial L}{\partial c^{(j)}}
$$

for each parameter $c^{(j)}$.

So, using Eq.(13) above, the $a$ term is

$$
\begin{gathered}
\frac{r(x)^{3 / 2}}{4 \pi}\left[\frac{d}{d x}\left(\frac{\partial L}{\partial a_{x}}\right)-\frac{\partial L}{\partial a}\right]=\frac{r(x)^{3 / 2}}{4 \pi} S[a] \\
=(r(x)-X)\left[a(x)-t_{0}^{\prime}(x)\right] .
\end{gathered}
$$

The $\theta(x)$ term is

$$
\begin{gathered}
\frac{r(x)^{5 / 2}}{2 \pi}\left[\frac{d}{d x}\left(\frac{\partial L}{\partial \theta_{x}}\right)-\frac{\partial L}{\partial \theta}\right]=\frac{r(x)^{5 / 2}}{2 \pi} S[\theta] \\
=[3 X-r(x)] r^{\prime}(x)-16 B^{2} x r(x)
\end{gathered}
$$

while the $t_{0}(x)$ term is

$$
\begin{gathered}
\frac{r(x)^{5 / 2}}{2 \pi}\left[\frac{d}{d x}\left(\frac{\partial L}{\partial t_{0}^{\prime}(x)}\right)-\frac{\partial L}{\partial t_{0}(x)}\right]=\frac{r(x)^{5 / 2}}{2 \pi} S\left[t_{0}\right] \\
=2 r(x) a^{\prime}(x)[r(x)-X] \\
-a(x)\left\{[r(x)-3 X] r^{\prime}(x)+16 B^{2} x r(x)\right\}
\end{gathered}
$$

The $r(x)$ term is

$$
\frac{r(x)^{9 / 2}}{\pi}\left[\frac{d}{d x}\left(\frac{\partial L}{\partial r_{x}}\right)-\frac{\partial L}{\partial r}\right]=\frac{r(x)^{9 / 2}}{\pi} S[r]
$$

$$
\begin{gathered}
=r(x)\left\{r ( x ) \left\{(3 X-r(x))\left[a^{2}(x)-2 a(x) t_{0}^{\prime}(x)-2 \theta^{\prime}(x)\right]\right.\right. \\
+12(B-1)\}+70 X\}-70 X^{2} .
\end{gathered}
$$

Using Eq.(4), we define the influence of any functional $R$ on parameter $c^{j}$ as:

$$
J\left[c^{j} ; R\right]=2 \operatorname{Re}\left(\int_{-\infty}^{\infty} R \frac{\partial \psi^{*}}{\partial c^{(j)}} d t\right)
$$

Once $R$ is given, we thus need to solve the four ODEs, $S\left[c^{j}\right]=$ $J\left[c^{j} ; R\right]$, with $c^{j}=\left\{a(x), t_{0}(x), r(x), \theta(x)\right\}$. 


\section{MORE EXAMPLES OF APPLICATION}

\section{A. Rogue waves of full Hirota equation}

The 'full' Hirota equation [41] is:

$$
R=i \alpha_{3}\left(\psi_{t t t}+6|\psi|^{2} \psi_{t}\right) \equiv R_{h}
$$

The right hand sides of the reduced dynamical system (4) for this equation take the form:

$$
\begin{gathered}
\frac{r(x)^{7 / 2}}{12 \pi \alpha_{3}} J\left[a ; R_{h}\right]=[X-r(x)] \times \\
\left\{r(x)\left[\left(a^{2}(x)-2\right) r(x)+4\right]-10 X\right\}
\end{gathered}
$$

and

$$
\begin{gathered}
\frac{r(x)^{9 / 2}}{2 \pi \alpha_{3} a(x)} J\left[r ; R_{h}\right]=210 X^{2}+r(x) \times \\
\left\{r(x)\left[a^{2}(x)(r(x)-3 X)+6(6-r(x)+3 X)\right]-210 X\right\},
\end{gathered}
$$

while $J\left[t_{0} ; R_{h}\right]=J\left[\theta ; R_{h}\right]=0$.

Setting $r(x)=1+4 B^{2} x^{2}$ reduces the dynamical system and leaves only the equation for $r$, viz. $S[r]=J\left[r ; R_{h}\right]$ to solve. It is straightforward to show that it can be solved with $a(x)=k$, an arbitary constant, $B=6 \alpha_{3} k+1$ and $\theta(x)=x c_{0}$ and $t_{0}=-v_{s} x$, where $c_{0}=k^{2}\left(c_{1}+c_{2} \alpha_{3} k\right)$ while

$$
v_{s}=\alpha_{3}\left(1-\frac{k^{2}}{2}\right) v_{1}-k
$$

Hence,

$$
\alpha_{3} k^{2}\left(2 c_{2}+v_{1}-2\right)-2 \alpha_{3}\left(v_{1}-6\right)+\left(2 c_{1}+1\right) k=0 .
$$

This equation must be valid for all $k$ and all $\alpha_{3}$. Consequently, we obtain $c_{1}=-\frac{1}{2}, v_{1}=6$ and $c_{2}=-2$. Then, the functions $t_{0}(x)$ and $\theta(x)$ in the trial function (12) become:

$$
\begin{aligned}
t_{0}(x) & =x\left[3 \alpha_{3}\left(k^{2}-2\right)+k\right], \\
\theta(x) & =-\frac{1}{2} k^{2} x\left(4 \alpha_{3} k+1\right) .
\end{aligned}
$$

This outcome agrees with the known exact result (with $\alpha_{2}=\frac{1}{2}$ ) found in [39]. The velocity factor, $t_{0}^{\prime}(x)$, can be zero for some combinations of $k$ and $\alpha_{3}$, thus, cancelling the effect of higherorder terms in the Hirota equation.

\section{B. Rogue wave of Lakshmanan - Porsezian - Daniel (LPD) equation}

The even higher-order Lakshmanan - Porsezian - Daniel (LPD) equation $[42,43]$ is:

$$
i \psi_{x}+\frac{1}{2} \psi_{t t}+|\psi|^{2} \psi=R[\psi(x, t)]
$$

where

$$
\begin{array}{r}
R[\psi(x, t)]=-\alpha_{4}\left(\psi_{t t t t}+6 \psi_{t}^{2} \psi^{*}+4 \psi\left|\psi_{t}\right|^{2}\right. \\
\left.+8 \psi_{t t}|\psi|^{2}+2 \psi_{t t}^{*} \psi^{2}+6 \psi|\psi|^{4}\right) \equiv R_{l} .
\end{array}
$$

For this equation, the right hand sides of the dynamical system (4) are:

$$
\begin{array}{r}
\frac{r(x)^{7 / 2}}{16 \pi a(x) \alpha_{4}} J\left[a ; R_{l}\right]=[r(x)-X]\{r(x) \times \\
\left.\left[\left(a^{2}(x)-6\right) r(x)+12\right]-30 X\right\}
\end{array}
$$

and

$$
\begin{aligned}
& \frac{r(x)^{13 / 2}}{2 \pi \alpha_{4}} J\left[r ; R_{l}\right]=2772 X^{3}+r(x) \times \\
& \left\{r ( x ) \left[12 a^{2}(x)(r(x)(r(x)[r(x)-3 X-6]\right.\right. \\
& \left.+35 X)-35 X^{2}\right)+a^{4}(x) r(x)^{2}[3 X-r(x)] \\
& -2 r(x)(3 r(x)[r(x)-3(X+4)]+70(3 X+2)) \\
& \left.+28 X(15 X+91)]-5040 X^{2}\right\}
\end{aligned}
$$

while $J\left[t_{0} ; R_{l}\right]=J\left[\theta ; R_{l}\right]=0$.

Again, setting $r(x)=1+4 B^{2} x^{2}$ reduces the system and leaves only the $r$ equation, $S[r]=J\left[r ; R_{l}\right]$ to solve. It is straightforward to show that it can be solved with $a(x)=$ $k$, an arbitrary constant, $B=\alpha_{4}\left(b_{1} k^{2}+b_{2}\right)+b_{0}, \theta(x)=$ $x\left\{3 \alpha_{4}\left[\left(k^{2}-2\right)^{2}+n_{2}\right]+k^{2} n_{1}\right\}$ and $t_{0}=-v_{e} x$, where $v_{e}=$ $k\left[\alpha_{4}\left(j_{1}+j_{2} k^{2}\right)-1\right]$.

Expanding $S[r]=J\left[r ; R_{l}\right]$, we readily find the constants, $b_{0}=$ $1, b_{1}=-12, b_{2}=12, j_{1}=-24, j_{2}=4, n_{1}=-1 / 2, n_{2}=-6$. Hence $B=1-12 \alpha_{4}\left(k^{2}-1\right)$, and

$$
\begin{aligned}
t_{0}(x) & =-v_{e} x=-k\left[4 \alpha_{4}\left(k^{2}-6\right)-1\right] x, \\
\theta(x) & =\left\{3 \alpha_{4}\left[\left(k^{2}-2\right)^{2}-6\right]-\frac{k^{2}}{2}\right\} x .
\end{aligned}
$$

This outcome also agrees with the known exact result (with $\alpha_{2}=\frac{1}{2}$ ) found in [39]. Again, the velocity factor, $t_{0}^{\prime}(x)$, is zero for some combinations of $k$ and $\alpha_{4}$.

\section{SOLITON UNDER INFLUENCE OF RAMAN DELAY}

The examples above are related to integrable equations in order for us to be able to compare the results of variational approach with exact solutions. However, the technique itself is fully justified only when there is no possibility of obtaining exact solutions. Thus, below, we consider more involved cases relevant to practical situations. Firstly, to show up the principle, we consider a soliton solution under the influence of Raman delay. This has been done earlier in $[44,45]$. Suppose, $R=\tau_{R} \psi\left(|\psi|^{2}\right)_{t} \equiv R_{r}$ is the only nonzero term in Eq.(2). Various approximations have been given to estimate the soliton shape for small delay, $\tau_{R}$, in this case [46-48]. The delay makes the soliton solution nonsymmetric in $t$, and this requires a new trial function. Here we take

$$
\psi(x, t)=\sqrt{\frac{Q(x)}{2 w(x)}} \operatorname{sech}\left(\frac{y_{1}}{w(x)}\right) \exp \left\{i\left[a(x) y_{1}-\theta(x)\right]\right\}
$$

where $Q(x)$ is the energy, $w(x)$ is the pulse width, and $y_{1}=$ $t-t_{0}(x)$, with $t_{0}(x)$ indicating the time delay offset.

The approximating procedure described in Section 1 leads to 5 ODEs. The $\theta(x)$ equation is:

$$
Q^{\prime}(x)=0,
$$

showing that $Q(x)=Q_{0}$ is a constant. The $a(x)$ equation is:

$$
Q(x)\left[a(x)-t_{0}^{\prime}(x)\right]=0
$$

showing that $a(x)=t_{0}^{\prime}(x)$. The $w(x)$ equation is:

$$
\frac{Q(x)}{6 w^{3}(x)}[w(x) Q(x)-2]
$$


showing that $w(x)=2 / Q_{0}=w_{0}$. Finally, the $t_{0}(x)$ equation is:

$$
\frac{8 \tau_{R}}{15 w_{0}^{4}}-t_{0}^{\prime \prime}(x)=0,
$$

showing that

$$
t_{0}^{\prime}(x)=a(x)=\frac{4 \tau_{R} Q(x)}{15 w_{0}^{3}} x=\frac{8 \tau_{R}}{15 w_{0}^{4}} x,
$$

as in [44] (where $w_{0}=1 / \eta, t_{0}=-\bar{t}$ and $a=-\omega$ ). Thus,

$$
t_{0}(x)=\frac{4 \tau_{R} x^{2}}{15 w_{0}^{4}}
$$

This result for time shift is in agreement with Eqs.(5b) and (13) in [45]. Plainly, $t_{0}(x)$ is a close analogue of vertical projectile motion under gravity, where the velocity $t_{0}^{\prime}(x)$ changes sign at $x=0$, but the acceleration, $t_{0}^{\prime \prime}(x)=\frac{8 \tau_{R}}{15 w_{0}^{4}}$, does not. This as expected, since the acceleration is caused by the Raman delay and this does not change sign.

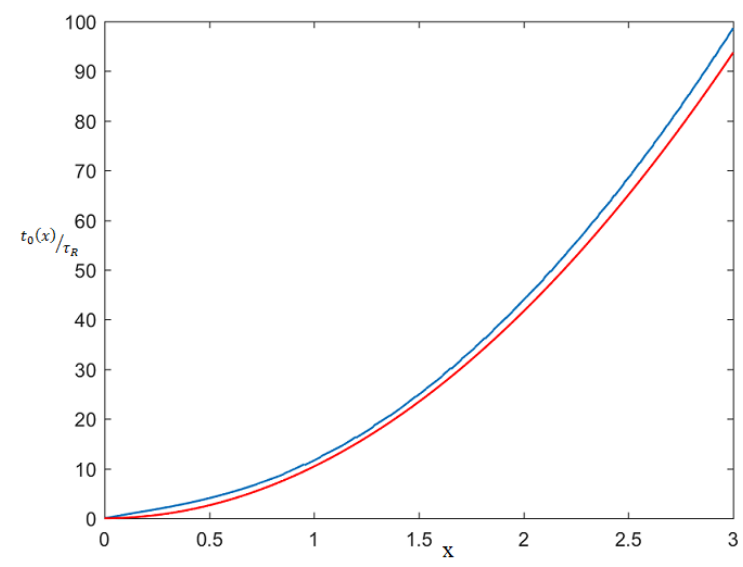

Fig. 1. Plot of the relative soliton offset, $t_{0} / \tau_{R}$, showing the effect of Raman delay. We compare the numerically-found result (blue curve) with the analytic result of Eq.(29), (red curve). Here, delay is given by $\tau_{R}=0.02$, and width $w_{0}=0.4$.

The remaining $Q(x)$ equation is:

$$
\frac{1}{w_{0}^{2}}+t_{0}^{\prime}(x)^{2}+2 \theta^{\prime}(x)=0
$$

showing that the rate of change of phase is $\theta^{\prime}(x)=-\frac{1}{2 w_{0}^{2}}-$ $\frac{1}{2}\left(\frac{8 \tau_{R} x}{15 w_{0}^{4}}\right)^{2}$, as in [44] (where $\theta=-\phi$ ). If $\tau_{R}=0$, we obtain

$$
\begin{array}{r}
\psi(x, t)=\frac{1}{w_{0}} \operatorname{sech}\left(\frac{t}{w_{0}}\right) \exp \left\{\frac{i x}{2 w_{0}^{2}}\right\} \\
=\frac{Q_{0}}{2} \operatorname{sech}\left(\frac{Q_{0} t}{2}\right) e^{\frac{1}{8} i Q_{0}^{2} x},
\end{array}
$$

which is the NLSE soliton of width $w_{0}$ with the energy $Q_{0}$. An example of the solution, showing the parabolic path, is given in Fig.2.

Our numerical propagation studies of this soliton, as exemplified in Fig.1, verify the above analytic results, and thus give confidence in the approach.

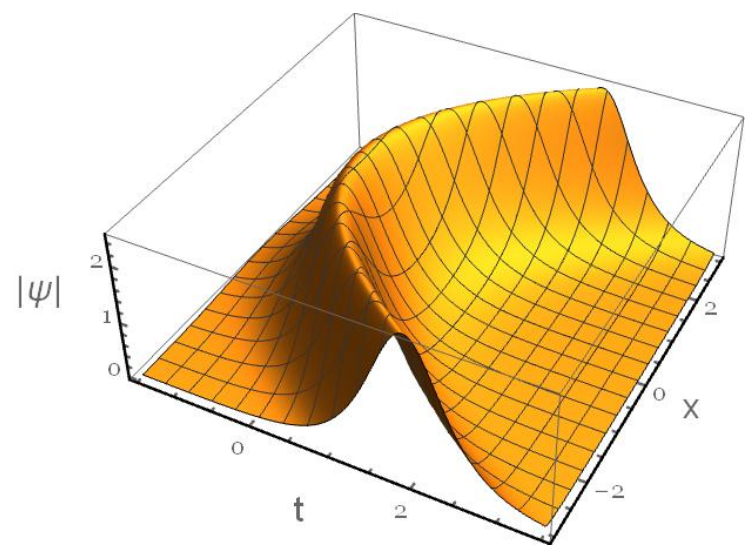

Fig. 2. Plot of the soliton, Eq.(23), under the effect of Raman delay, using Eqs.(24) to (30). Here, delay is given by $\tau_{R}=0.02$, and width $w_{0}=0.4$. At the ends $(x= \pm 3)$, the $t$ offset is about 1.8 .

\section{ROGUE WAVE UNDER INFLUENCE OF RAMAN DE- LAY}

Now, instead of a soliton, we consider a rogue wave solution which is localized in time. For this, we use the same trial function as in Eqs. $(7,8)$ and $(9)$.

The ODEs will naturally differ from the previous cases. The $\theta(x)$ equation is:

$$
r(x)\left[r^{\prime}(x)+16 x\right]=3\left(4 x^{2}+1\right) r^{\prime}(x) .
$$

To solve it, we set $z=\sqrt{X}=\sqrt{1+4 x^{2}}$ and $r(z)=s^{2}(z)$. This gives:

$$
\left[3 z^{2}-s^{2}(z)\right] s^{\prime}(z)=2 z s(z) .
$$

We find that the solution is:

$$
s(z)=\frac{1}{6 c}\left(F^{1 / 3}+F^{-1 / 3}-1\right)
$$

where

$$
F=54 c^{2} z^{2}-1+6 c z \sqrt{3} \sqrt{27 c^{2} z^{2}-1},
$$

where $c$ is an arbitrary real solution parameter.

Note that this remains real even when $c$ is small. In fact $\lim _{c \rightarrow 0} s(z)=z$, so $\lim _{c \rightarrow 0} r(z)=z^{2}$. Also, $r(x)$ is even in c, so we only need to use $c \geq 0$. This means that $r(0) \leq 1$ for any c. We plot the function $r(x)$ in Fig.3.

Since $c$ is generally small $(c<0.1)$, it is convenient to expand this result. For $c \geq 0$ :

$$
r(z)=z^{2}\left(1-2 c z+6 c^{2} z^{2}-21 c^{3} z^{3}+80 c^{4} z^{4}+\cdots\right) .
$$

i.e.

$$
\begin{aligned}
& r(x)=\left(1+4 x^{2}\right)\left[1-2 c \sqrt{1+4 x^{2}}+6 c^{2}\left(1+4 x^{2}\right)\right. \\
& \left.-21 c^{3}\left(1+4 x^{2}\right)^{3 / 2}+80 c^{4}\left(1+4 x^{2}\right)^{2}+\cdots\right] .
\end{aligned}
$$

So, when $c=0$, the solution reduces to the known NLSE result, $r(x)=\left(1+4 x^{2}\right)$. In solving the 4 ODEs, we take $c$ to be arbitrary but larger than $\tau_{R}$.

Using, Eq.(4) with $S\left[t_{0}\right]$ from Eq.(17) and $J\left[t_{0} ; R_{r}\right]$ from Eq.(19), for $t_{0}(x)$, we find:

$$
\frac{r(x)^{9 / 2}}{32 \pi} J\left[t_{0} ; R_{r}\right]=\tau_{R}\left\{r(x)[5 X-r(x)]-7 X^{2}\right\},
$$




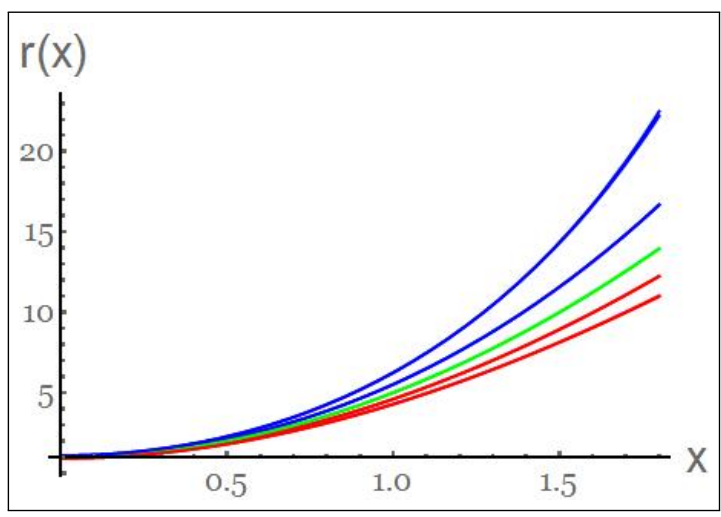

Fig. 3. Plot of function $r(x)$ for various c values. The blue curves are for $c<0$. This solution corresponds to the expansion in Eq.(37) with $c=-0.04$ (top blue curve) and $c=-0.02$ (next blue curve). The green curve, viz.. $r(x)=z^{2}=1+4 x^{2}$ is for $c=0$. The two red curves are plots of Eqs.(35) and (36). The upper red curve is for $c=0.02$. The lower red curve is for $c=0.04$. In each case, the expansion, Eq.(37), is superimposed on the exact result. The curves are indistinguishable.

so the $t_{0}(x)$ equation is:

$$
\begin{gathered}
16 \tau_{R}\left\{r(x)[r(x)-5 X]+7 X^{2}\right\} \\
+r(x)^{2}\left\{2 r ( x ) \left(a^{\prime}(x)[r(x)-X]\right.\right. \\
\left.-8 x a(x))+a(x)[3 X-r(x)] r^{\prime}(x)\right\}=0,
\end{gathered}
$$

where $X=1+4 x^{2}$. The other r.h.s. factors, $J\left[r ; R_{r}\right], J\left[a ; R_{r}\right], J\left[\theta ; R_{r}\right]$ are zero.

The $r(x)$ equation is:

$$
\begin{gathered}
r(x)\left\{r ( x ) [ r ( x ) - 3 X ] \left[a^{2}(x)\right.\right. \\
\left.\left.-2 a(x) t_{0}^{\prime}(x)-2 \theta^{\prime}(x)\right]-70 X\right\}+70 X^{2}=0 .
\end{gathered}
$$

and the $a(x)$ equation is:

$$
[X-r(x)]\left[a(x)-t_{0}^{\prime}(x)\right]=0
$$

Now, if we take $r(x)=4 x^{2}+1$ to solve the last equation, then the 2-nd one cannot be solved. So, we need to take $a(x)=t_{0}^{\prime}(x)$ to solve it. This reduces Eq.(39) to:

$$
\begin{aligned}
& r(x)\left\{r ( x ) \left[-t_{0}^{\prime}(x)\left((r(x)-3 X) r^{\prime}(x)\right.\right.\right. \\
& +16 x r(x))+2 r(x)(r(x)-X) t_{0}^{\prime \prime}(x) \\
& \left.\left.\quad+16 \tau_{R}\right]-80 \tau_{R} X\right\}+112 \tau_{R} X^{2}=0
\end{aligned}
$$

and it reduces Eq.(40) to:

$$
\begin{aligned}
r(x)\left\{r(x)[3 X-r(x)]\left[t_{0}^{\prime}(x)^{2}+2 \theta^{\prime}(x)\right]\right. & \\
-70 X\}+70 X^{2} & =0 .
\end{aligned}
$$

We can solve the resultant ODEs, i.e. Eqs.(33), (42) and (43), to various orders in $\tau_{R}$. We take $c$ and $\tau_{R}$, to be small and have similar order (around $10^{-3}$ ). We assume that the offset, $\mathrm{c}$, is not much smaller than $\tau_{R}$, so we cannot have $\tau_{R} / c>>1$. This excludes $c=0$. In fact, we expect $c>\tau_{R}$.

Using Eq.(38), we have:

$$
r(x)=X\left[1-2 c X^{1 / 2}+6 c^{2} X-21 c^{3} X^{3 / 2}\right.
$$

$$
\left.+80 c^{4} X^{2}+\cdots\right]
$$

Then:

$$
\begin{aligned}
a(x)= & t_{0}^{\prime}(x)=\frac{2 \tau_{R} x}{c X^{3 / 2}}\left[2\left(8 x^{2}+3\right)+33 c X^{1 / 2}\right. \\
& \left.+215 c^{2} X\right]+33 \tau_{R} \arctan (2 x) .
\end{aligned}
$$

Now, taking $t_{0}(0)=0$ we obtain:

$$
\begin{gathered}
t_{0}(x)=\tau_{R}\left\{\frac{1}{c}\left(\frac{8 x^{2}+1}{X^{1 / 2}}-1\right)\right. \\
\left.+33 x \arctan (2 x)+\frac{215}{2} c\left[X^{1 / 2}-1\right]\right\} .
\end{gathered}
$$

So, for small $x$, we have

$$
\begin{aligned}
\frac{t_{0}(x)}{\tau_{R}} & \approx\left(215 c+\frac{6}{c}+66\right) x^{2} \\
& -\left(215 c+\frac{10}{c}+88\right) x^{4}+\cdots
\end{aligned}
$$

In the soliton case, we also found that $t_{0}(x)$ varied as $\tau_{R} x^{2}$. If $c$ is small, say $c<0.1$, then:

$$
t_{0}(x) \approx 6 \frac{\tau_{R}}{c} x^{2}\left(1-\frac{5}{3} x^{2}+\cdots\right)
$$

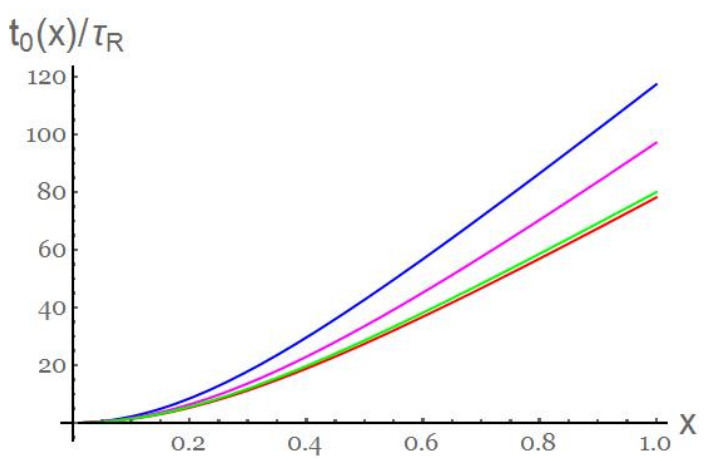

Fig. 4. Plot of function $t_{0}(x) / \tau_{R}$, from Eq.(46) for various $c$ : $c=0.04$ (blue curve), $c=0.4$ (magenta), $c=0.2$ (red), and $c=0.1$ (green). tion:

Hence, we obtain the following simple form for the accelera-

$$
\begin{gathered}
\frac{a^{\prime}(x)}{\tau_{R}}=\frac{t_{0}^{\prime \prime}(x)}{\tau_{R}}= \\
\frac{2}{c}\left[6 X^{-5 / 2}+66 c X^{-2}+215 c^{2} X^{-3 / 2}\right] .
\end{gathered}
$$

Thus, the acceleration, $t_{0}^{\prime \prime}(x)$ of the ridge takes the same sign as that for the soliton case found in Eq.(27), and is also an even function, but it has a more complicated form. As for the soliton case, it is proportional to $\tau_{R}$. The estimates for the function $t_{0}(x)$ are shown in Fig. 5 along with the results of numerical simulations for the same set of parameters. As can be seen from this figure, numeric solution of the 4 ODEs give results which are reasonably close to the above approximate solutions for small $\tau_{R}$, say $\tau_{R}<0.002$.

The Raman effect causes a delay, so we expect deceleration. It is analogous to the deceleration found for the Raman soliton 


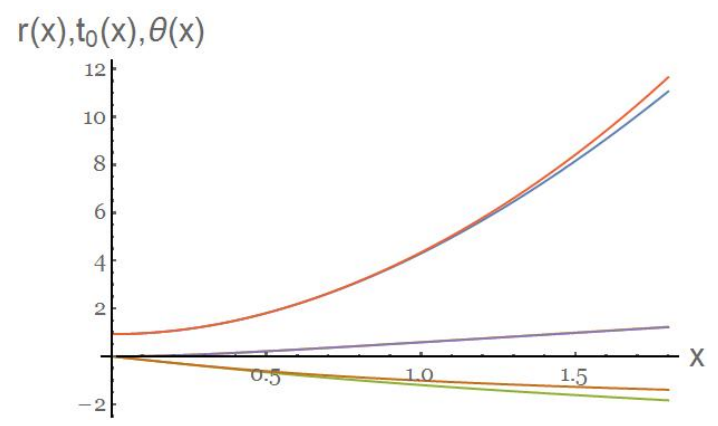

Fig. 5. Plot of functions $t_{0}(x)$, Eq.(46), $r(x)$, using the results of section 6, including Eq.(38), and $\theta(x)$, using Eq.(53), together with the same functions found numerically by solving Eqs.(33) to (41). Here, $c=0.04$, and the delay is given by $\tau_{R}=0.005$. The two upper curves give $r(x)$, while the two lower positive ones give $t_{0}(x)$ and the two negative ones show $\theta(x)$. In each case, the exact numeric solution and the approximation for it is very close.

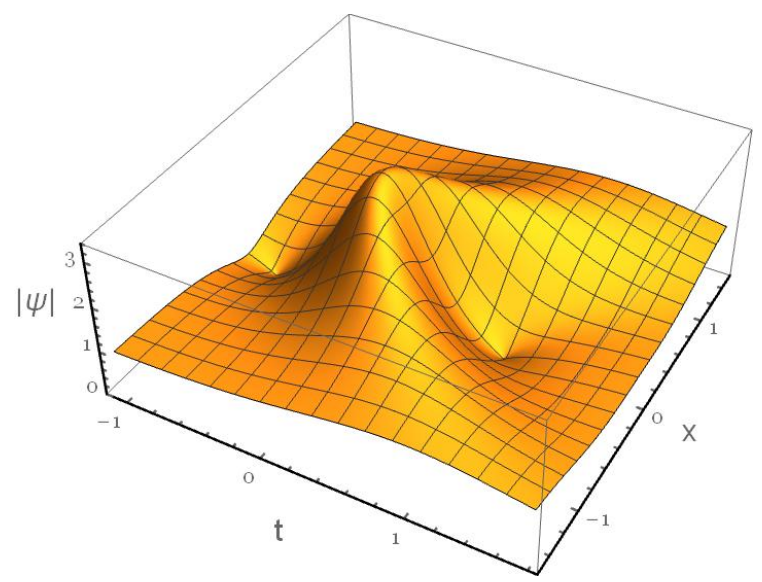

Fig. 6. Plot of the rogue wave, Eq.(7), using Eqs.(46) to (53) for delay given by $\tau_{R}=0.005$. Here $c=0.04$.

in Eq.(28). Examples of evolution are shown in Figs.6 and 7. Acceleration here is clearly seen.

Now, $\frac{t_{0}^{\prime \prime}(0)}{\tau_{R}}=\frac{2}{c}\left(6+66 c+215 c^{2}\right)$, and we define this to be $c_{0}$. On plotting the acceleration, we see that it plainly has a gaussian-type form. We find that it can be approximated by:

$$
\frac{t_{0}^{\prime \prime}(x)}{\tau_{R}} \approx c_{0} e^{-6 x^{2}}
$$

Integrating shows that:

$$
\frac{t_{0}^{\prime}(x)}{\tau_{R}} \approx c_{0} \sqrt{\frac{\pi}{6}} \operatorname{erf}(\sqrt{6} x),
$$

where 'erf' indicates error function. So the final expression for velocity is $t_{0}^{\prime}( \pm \infty) / \tau_{R} \approx \pm \frac{c_{0}}{2} \sqrt{\frac{\pi}{6}}$. Then

$$
\frac{t_{0}(x)}{\tau_{R}} \approx \frac{c_{0}}{2} \sqrt{\frac{\pi}{6}}\left[x \operatorname{erf}(\sqrt{6} x)-\frac{1-e^{-6 x^{2}}}{\sqrt{6 x}}\right] .
$$

The comparisons in Fig.8 show that this is a convenient and accurate approximation, giving a simple view of the ridge offset during propagation.

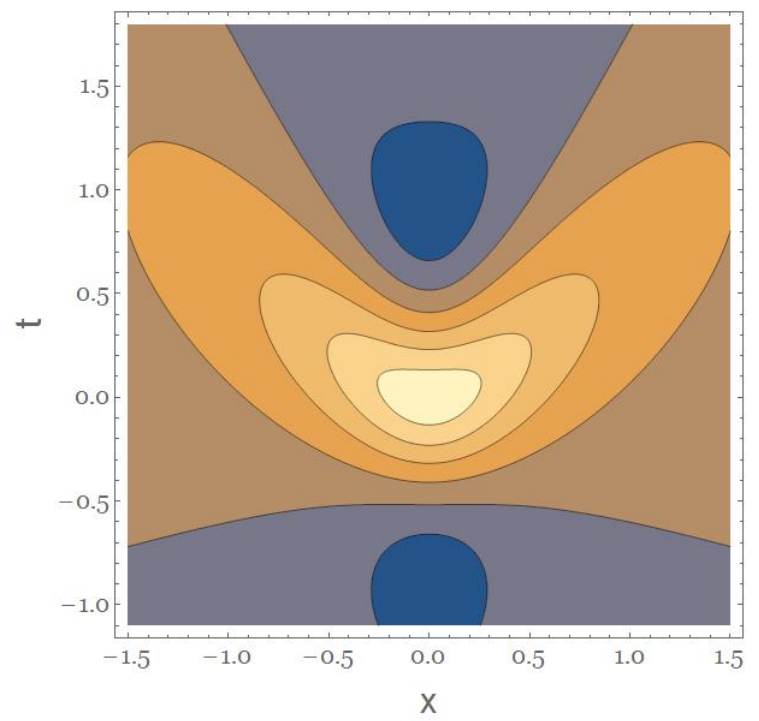

Fig. 7. Contour plot of the rogue wave, Eq.(7), using Eqs.(46) to (53) for delay given by $\tau_{R}=0.005$. Here $c=0.04$ and the propagation direction, $x$, is the horizontal axis, while the transverse axis, $t$, is vertical.

So, for small $\mathrm{x}$,

$$
\frac{t_{0}(x)}{\tau_{R}} \approx \frac{c_{0}}{2} x^{2}\left[1-x^{2}+\frac{6 x^{4}}{5} \cdots\right]
$$

For $c_{0}=\frac{2}{c}\left(6+66 c+215 c^{2}\right)$, we find that $c_{0}$ reaches its minimum of 275 when $\mathrm{c}=0.17$, and $275<c_{0}<295$ for $0.1<c<0.28$. For $c_{0}=\frac{2}{c}(6+66 c)$, we find that $c_{0}=250$ when $\mathrm{c}=0.1$, and then $c_{0}$ decreases monotonically as c increases. Now $t_{0}(x) \approx \frac{c_{0}}{2} \tau_{R} x^{2}$, so we need $\tau_{R}<c$ to get realistic [not too high]. For typical values of $c$, say $c=0.2$, we have $c_{0}$ around 250 , and hence

$$
t_{0}(x) \approx 125 \tau_{R} x^{2}
$$

for small $\mathrm{x}$.

Eqs.(46) to (48) are valid for all $x$; thus position $t_{0}(x)$ and acceleration $t_{0}^{\prime \prime}(x)$ are even functions of $x$, while velocity $t_{0}^{\prime}(x)$ is an odd function in $x$. It thus corresponds to a more complicated form than the motion under gravity of section 6 , as here the analogous particle would be subject to a force prorportional to $\frac{2 \tau_{R}}{c}\left[6 X^{-5 / 2}+66 c X^{-2}+215 c^{2} X^{-3 / 2}\right]$. From Eq.(49), this force varies roughly as $c_{0} \tau_{R} e^{-6 x^{2}}$. It approaches zero for large $|x|$ but does not change sign.

Now, let us turn to the exponent term $\theta(x)$. Setting $\theta(0)=0$, we have:

$$
\theta(x)=-\frac{35 c}{2} \operatorname{arcsinh}(2 x)
$$

Expansion

$$
\theta(x)=-35 c x\left(1-\frac{2}{3} x^{2}+\cdots\right),
$$

agrees with the results from the PDE simulations. Now

$$
\theta^{\prime}(x)=-\frac{35 c}{\sqrt{X}}
$$

where $X=1+4 x^{2}$. Plainly, this is almost constant for small $x$. 


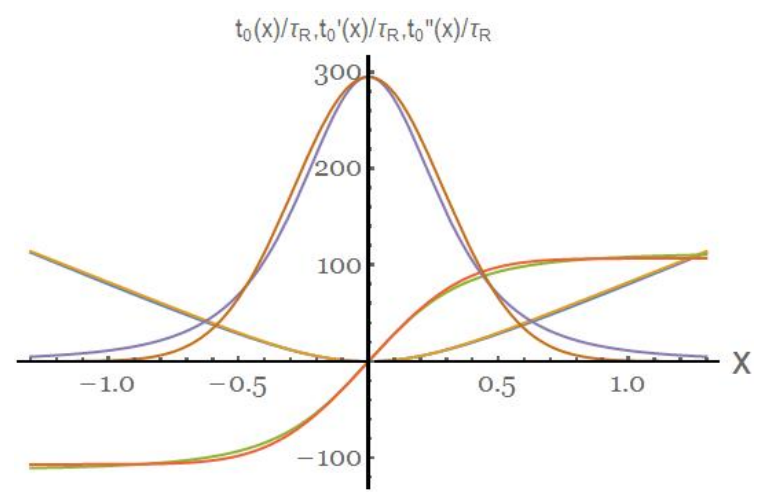

Fig. 8. Plot of functions $t_{0}^{\prime \prime}(x) / \tau_{R}$ (even function of $\mathrm{x}$ with $t_{0}(0) \approx c_{0}=300$ here), $t_{0}^{\prime}(x) / \tau_{R}$ (odd function of $\mathrm{x}$ ) and $t_{0}(x) / \tau_{R}$ (even function of $x$ with $t_{0}(0)=0$ ) from original Eqs.(46), (45) and (48), respectively, together with a comparison with the gaussian-based approximations for them, given by Eqs.(49), (50) and (51), all for $c=0.1$. Here, $c_{0}=\frac{2}{c}\left(6+66 c+215 c^{2}\right)$.

In the next section, we plot $t_{0}(x) / \tau_{R}$ for various $c$ on the same diagram. We also plot $\theta(x) / c$ and $\left(r(x)-1-4 x^{2}\right) / c$ to estimate the next term in $r(x)$.

\section{COMPARISON WITH NUMERICAL SIMULATIONS OF PDE}

For $x=0$, we have $a(x)=\theta(x)=0$, so $\psi$ is real. It maximum value, $\psi_{m}$ occurs when $t=0$, and is given by $\psi_{m}=\frac{4}{r(0)}-$ $1 \approx 3+8 c$. As noted earlier, this is a function of $c$ only, as $r(0)=1-2 c$. Using the model, at fixed $x$, the intensity, $\mathrm{I}$, is:

$$
I=\frac{\left\{r(x)+4\left[t-t_{0}(x)\right]^{2}-4\right\}^{2}+64 x^{2}}{\left(r(x)+4\left[t-t_{0}(x)\right]^{2}\right)^{2}}
$$

We note that $r(x)$ is close to $1+4 x^{2}$ for small c. By setting $\frac{\partial I}{\partial t}=0$, we find that there are 3 stationary points. The first is at $t=t_{0}(x)$, and it is a maximum since $\frac{\partial^{2} I}{\partial t^{2}}<0$, as $4+16 x^{2}>>r(x)$. On the either side, we have $t=t_{0}(x) \pm \frac{1}{2} \sqrt{16 x^{2}+4-r(x)}$. These are minima, since $\frac{\partial^{2} I}{\partial t^{2}}>0$ for each.

In the simulations of the PDE we can start with initial conditions with $r(0)$ close to 1 and $t_{0}(0)=\theta(0)=0$. At each $\mathrm{x}$, the value of $t_{0}$ giving the maximum intensity is then labelled as $t_{0}(x)$. This shows up the dependence of $t_{0}(x)$ on $\mathrm{p}$. At this point, we use the intensity maximum, $I_{m}=\frac{[r(x)-4]^{2}+64 x^{2}}{r(x)^{2}}$, to find $r(x)$ by solving the quadratic equation. This shows up the dependence of $r(x)$ on $\mathrm{p}$. At each such point, we have $\mathrm{x}$ and $r(x)$, and the complex number $\psi\left(x, t=t_{0}(x)\right)=\left[\frac{4(1+2 i x)}{r(x)}-1\right] e^{i[x-\theta(x)]}$, we directly find $\theta(x)$. So we can compare the PDE results with the predicted results found by solving the 4 ODEs approximately. Using the numerical PDE runs, we now find how $r(x), t_{0}(x)$ and $\theta(x)$ depend on $c$ and $\tau_{R}$.

From numerics, we can plot $r(x)-1-4 x^{2}$ for various values of $c$, and then observe if it can be expressed as $c^{n} f(x)$ for some integer $n$, i.e. whether the dependence on $c$ and $x$ can be separated for a suitably chosen value of $\mathrm{n}$. Results show that $n=1$ with $f(x)=-2\left(1+4 x^{2}\right)^{k}$ works well for $k=1.865$, thus roughly supporting the result of Eq.(38), which had $k=3 / 2$.
Here, c is arbitrarily chosen, and it determines how much $r(0)$ differs from 1.

Similarly, we can plot $t_{0}(x) / \tau_{R}$ for various values of $c$, and then observe if it can be expressed as $j[c] g(x)$, and plot $\theta(x)$ for various values of $c$, and then observe if it can be expressed as $\operatorname{ch}(x)$. For small $\mathrm{x}$, we find that $t_{0}(x) / \tau_{R} \equiv x^{2}$, agreeing with the analytic form.

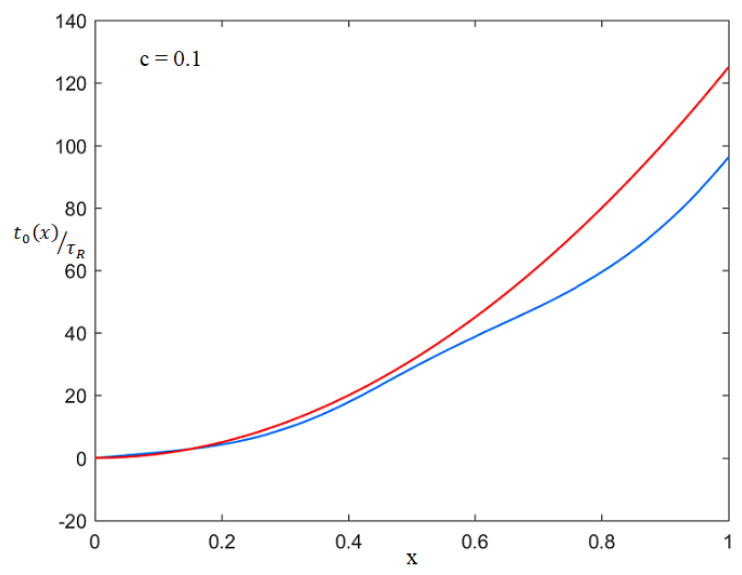

Fig. 9. Plot of function $t_{0}(x) / \tau_{R}$, from numerics (blue curve) for $c=0.1$. The magenta curve is from the analytic prediction of Eq.(52). It is in fairly good agreement with the numeric curve. Thus, the reduced model has provided a reasonably good description of the rogue wave dynamics.

For small $c$, the numerics shows that:

$$
t_{0}(x) \approx 25 \tau_{R} x^{2}(1+14.7 c),
$$

for offset c. So, when $c$ is not too small, $t_{0}(x)$ is of the same order as the analytic form, Eq.(52), found earlier. An example, with $c=0.1$ is given in Fig.9.

\section{NUMERICAL DETERMINATION OF FUNCTIONS}

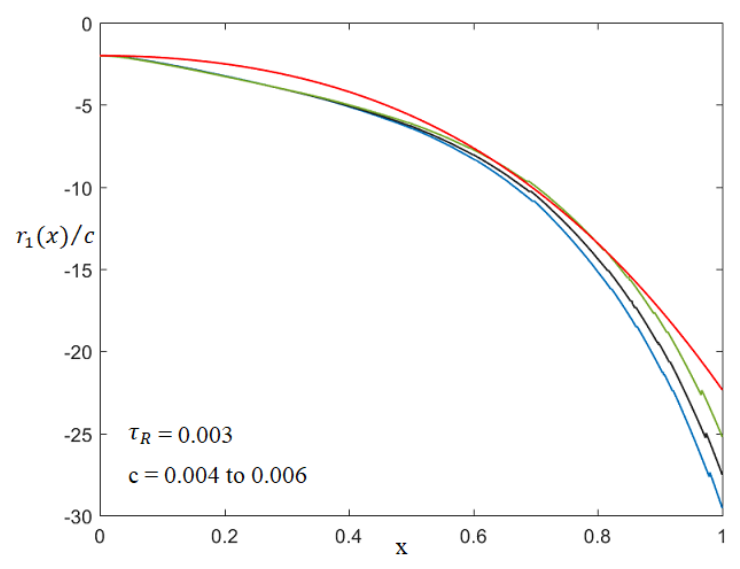

Fig. 10. The function $r_{1}(x)$ found from numerical solution of the PDE. Three values of $c$, from (blue curve) 0.004 to (green curve) 0.006 are used and the initial value is $r(0)=1-2 c$. The analytic form, $r_{1}(x)=\left(r(x)-1-4 x^{2}\right) / c \approx-2\left(1+4 x^{2}\right)^{3 / 2}$, shown by red curve is close to the numerical results. 
Suppose $\theta(x)=-c^{n} y(x)$ and $r(x)=4 x^{2}+1+c^{n} r_{1}(x)$ for some unknown $\mathrm{n}$. Then we find, in this model, that, on the 'ridge' formed by the maxima at each $\mathrm{x}$ :

$$
\begin{gathered}
\phi\left[x, t=t_{0}(x) ; c\right]= \\
\left(-1+\frac{4(1+2 i x)}{c^{n} r_{1}(x)+4 x^{2}+1}\right) e^{i\left(x+c^{n} y(x)\right)}
\end{gathered}
$$

while NLSE result is:

$$
\phi[x, t=0]=\left(-1+\frac{4(1+2 i x)}{4 x^{2}+1}\right) e^{i(x)}
$$

Using numerics, we subtract one from the other. Suppose, the difference $\Delta$ is:

$$
\Delta=-c^{n} e^{i x}[f(x)+i n(x)] .
$$

Along the 'ridge', we measure this $\Delta$ as a complex function of $\mathrm{x}$. So, by finding a few values of $\Delta$, we determine the dependence on $c$, and immediately find the value of $n$, which turns out to be 1.

Then, expanding $e^{i x}\left(\phi\left[x, t=t_{0}(x) ; c\right]-\phi[x, t]-\Delta\right)$ for small $c$ gives the first non-zero term. Equating it to zero gives

$$
f(x)+i n(x)+\frac{\frac{4 i r_{1}(x)}{i-2 x}+(1-2 i x)(2 x-3 i) y(x)}{(2 x+i)^{2}}=0 .
$$

Solving this equation, we obtain $r_{1}(x), y(x)$ explicitly:

$$
r_{1}(x)=\frac{1}{12}\left(4 x^{2}+1\right)\left[\left(3-4 x^{2}\right) f(x)+8 x n(x)\right]
$$

and

$$
y(x)=\frac{1}{3}[2 x f(x)-n(x)] .
$$

We use this procedure to produce curves for $r_{1}(x)$ and $y(x)$ for various values of $c$. From Fig. 10 and curves for other $c$ values, we find $r_{1}(x) \approx-2\left(4 x^{2}+1\right)^{1.865}$. This compares with $-2\left(4 x^{2}+1\right)^{3 / 2}$ found from the analytic result, so the approximate result is reasonably accurate.

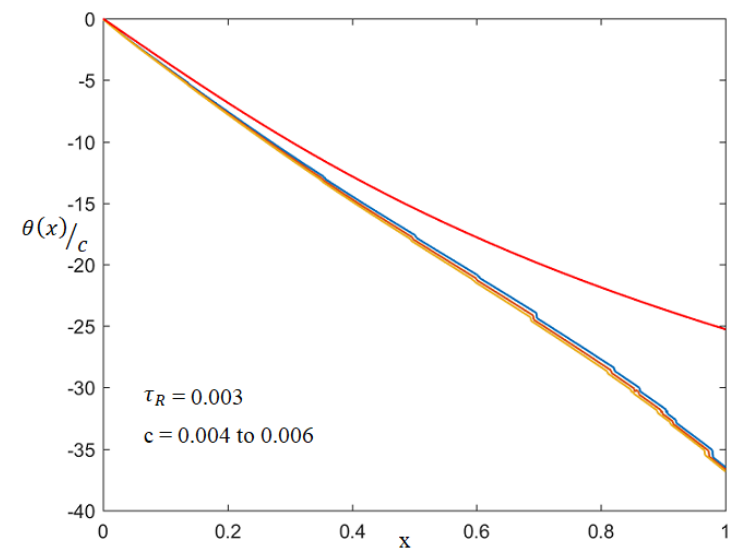

Fig. 11. Numerical PDE solution giving $\theta(x) / c$. Various values of $c$ are used and the initial value is $r(0)=1-2 c$. The analytic result is plotted as the magenta line, $\theta(x) / c=$ $-(35 / 2) \operatorname{arcsinh}(2 x) \approx-35 x$ for $x$ small, using Eq.(53). The numerically found curve for $\theta(x) / c$ is quite close to $-35 x$ over this range, $0<x<1$.
From Fig. 11 and curves for other $c$ values, we find $y(x) \approx 35 x$ and hence $\theta(x) \approx-35 c x$ for $x<1$. For small $\mathrm{x}$, we find:

$$
y(x) \equiv-\theta(x) /(c)=35 x+2\left(96+\frac{35}{3}\right) x^{3}+\cdots .
$$

Plainly, for small $\mathrm{x}$, which is the main region of interest for the localized rogue wave, we have $\theta(x) \approx-35 c x$, which is in good agreement with the value from the expression found numerically.

\section{CONCLUSIONS}

In this work, we have found rogue wave solutions of higherorder extensions of the NLSE. Our approach can be useful when exact solutions of the evolution equation cannot be found. As an example, approximate solutions in the form of rogue waves have been obtained and analyzed for an optical fiber with a Raman delay term. Based on this example, the technique can be applied to other higher-order terms in the extended NLSE.

In order to confirm the applicability of the technique, we tested it on a few integrable equations and compared the results with the exact solutions. The equation with the Raman term does not have the exact solution in the form of a rogue wave. In this case, we conducted numerical simulations confirming that the approximate solutions are sufficiently accurate. The approach presented here could also be used to study other optical systems that are only described by non-integrable equations, for example, ultrafast nonlinear quadratic systems.

Rogue waves may be difficult to detect with existing equipment in optics. However, new techniques have been developed recently that may help in such measurements [49]. Namely, time stretching analog-to-digital converters [50,51], time microscopes [52] and time lens magnifiers [53] may be able to resolve individual ultra-short pulses that could not be done with traditional pulse accumulating methods. In this sense, theory and experiment are progressing equally well, and this may allow us to witness experimental observations of Raman rogue waves soon. The first experimental observation, using single shot technique, has already been made in [12]. Fine details of the pulse profile that we studied here could also be observed in the near future.

\section{FUNDING INFORMATION}

The Australian Research Council (Discovery Projects DP140100265, DP150102057 and DE1312345); Volkswagen Stiftung.

The authors thank Wonkeun Chang for assistance.

\section{REFERENCES}

1. D. R. Solli, C. Ropers, P. Koonath \& B. Jalali, Optical rogue waves, Nature, 450, 1054 (2007)

2. J. M. Soto-Crespo, Ph. Grelu and N. Akhmediev, Dissipative rogue waves: extreme pulses generated by passively mode-locked lasers, Phys. Rev. E, 84, 016604 (2011).

3. A. Zaviyalov, O. Egorov, R. lliew and F. Lederer, Rogue waves in mode-locked fiber lasers, Phys. Rev. A 85, 013828 (2012).

4. M. G. Kovalsky, A. A. Hnilo, and J. R. Tredicce, Extreme events in the Ti: sapphire laser, Opt. Lett., 36, 4449 (2011).

5. C. Lecaplain, Ph. Grelu, J. M. Soto-Crespo and N. Akhmediev, Dissipative Rogue Waves Generated by Chaotic Pulse Bunching in a Mode-Locked Laser, Phys. Rev. Lett., 108, 233901 (2012).

6. C. Lecaplain, Ph. Grelu, J. M. Soto-Crespo and N. Akhmediev, Dissipative rogue wave generation in multiple-pulsing mode-locked fiber laser, J. Optics, 15, 064005 (2013). 
7. S. A. Kolpakov, H. Kbashi, and S. V. Sergeyev, Dynamics of vector rogue waves in a fiber laser with a ring cavity, Optica, 3, 870 (2016).

8. M. Tlidi, K. Panajotov, M.I Ferré, and M. G. Clerc, Drifting cavity solitons and dissipative rogue waves induced by time-delayed feedback in Kerr optical frequency comb and in all fiber cavities, Chaos, 27, 114312 (2017); doi.org/10.1063/1.5007868

9. S. Residori, Bortolozzo, A. Montina, F. Lenzini and F. T. Arecchi, Rogue waves in spatially extended optical systems, Fluctuat. Noise Lett., 11. 1240014 (2012).

10. A. Montina, U. Bortolozzo, S. Residori and F. T. Arecchi, Non-Gaussian statistics and extreme waves in a nonlinear optical cavity, Phys. Rev. Lett., 103. 173901 (2009).

11. P.-H. Hanzard, M. Talbi, D. Mallek, A. Kellou, H. Leblond, F. Sanchez, T. Godin, and A. Hideur, Brillouin scattering-induced rogue waves in self-pulsing fiber lasers, Scientific Reports 7, 45868 (2017).

12. A. F. J. Runge, C. Aguergaray, N. G. R. Broderick, and M. Erkintalo, Raman rogue waves in a partially mode-locked fiber laser, Opt Lett., 39, 319-322 (2014)

13. J. M. Dudley, F. Dias, M. Erkintalo and G. Genty, Instabilities, breathers and rogue waves in optics, Nature Photonics, 8, 755 - 764 (2014).

14. M. Onorato, S. Residori, U. Bortolozzo, A. Montina, and F. Arecchi, Rogue waves and their generating mechanisms in different physical contexts, Phys. Rep., 528, $47-89$ (2013).

15. N. Akhmediev, B. Kibler, F. Baronio, M. Belić, W.-P. Zhong, Y. Zhang, W. Chang, J. M. Soto-Crespo, P. Vouzas, P. Grelu, C. Lecaplain, K. Hammani, S. Rica, A. Picozzi, M. Tlidi, K. Panajotov, A. Mussot, A. Bendahmane, P. Szriftgiser, G. Genty, J. Dudley, A. Kudlinski, A. Demircan, U. Morgner, S. Amiraranashvili, C. Bree, G. Steinmeyer, C. Masoller, N. G. R. Broderick, A. F. J. Runge, M. Erkintalo, S. Residori, U. Bortolozzo, F. T. Arecchi, S. Wabnitz, C. G. Tiofack, S. Coulibaly and M. Taki, Roadmap on optical rogue waves and extreme events, J. Opt., 18, 063001 (2016).

16. W. Chang, J. M. Soto-Crespo, P. Vouzas and N. Akhmediev, Extreme soliton pulsations in dissipative systems, Phys. Rev. E, 92, 022926 (2015).

17. W. Chang, J. M. Soto-Crespo, P. Vouzas and N. Akhmediev, Extreme amplitude spikes in a laser model described by the complex Ginzburg - Landau equation, Opt. Lett., 40, 2949 (2015).

18. N. Akhmediev, A. Ankiewicz and M. Taki, Waves that appear from nowhere and disappear without a trace, Phys. Lett. A 373, 675 (2009).

19. N. Akhmediev, J. M. Soto-Crespo and A. Ankiewicz, Extreme waves that appear from nowhere: on the nature of rogue waves, Phys. Lett. A, $373,2137-2145$ (2009).

20. A. Ankiewicz, J. M. Soto-Crespo and N. Akhmediev, Rogue waves and rational solutions of the Hirota equation, Phys. Rev. E, 81, 046602 (2010).

21. U. Bandelow and N. Akhmediev, Persistence of rogue waves in extended nonlinear Schrödinger equations: Integrable Sasa - Satsuma case, Phys. Lett. A, 376, 1558 (2012).

22. F. Baronio, A. Degasperis, M. Conforti, and S. Wabnitz, Solutions of the Vector Nonlinear Schrödinger Equations: Evidence for Deterministic Rogue Waves, Phys. Rev. Lett., 109, 044102 (2012).

23. Chao-Qing Dai, Jiu Liu, Yan Fan and Ding-Guo Yu, Two-dimensional localized Peregrine solution and breather excited in a variable-coefficient nonlinear Schrödinger equation with partial nonlocality, Nonlinear Dyn., (2017), 88, 1373-1383. DOI 10.1007/s11071-016-3316-x.

24. Yue-Yue Wang, Chao-Qing Dai, Guo-Quan Zhou, Yan Fan and Liang Chen, Rogue wave and combined breather with repeatedly excited behaviors in the dispersion/diffraction decreasing medium, Nonlinear Dyn., (2017),87, 67-73 DOI 10.1007/s11071-016-3025-5

25. Ji-tao Li, Xian-tu Zhang, Ming Meng, Quan-tao Liu, Yue-yue Wang and Chao-qing Dai, Control and management of the combined Peregrine soliton and Akhmediev breathers in PT -symmetric coupled waveguides, Nonlinear Dyn., (2016), 84, 473-479 DOI 10.1007/s11071-0152500-8

26. D. Anderson, Variational approach to nonlinear pulse propagation in optical fibers, Phys. Rev., A 27, 3135 (1983).

27. A. I. Maimistov, Evolution of solitary waves which are approximately solitons of a nonlinear Schrödinger equation, J. Exp. Theor. Phys., 77, 727 (1993) [Zh. Eksp. Teor. Fiz. 104, 3620 (1993), in Russian].

28. A. Ankiewicz, N. Akhmediev, N. Devine, Dissipative solitons with a Lagrangian approach (Invited paper), Opt. Fibre Techn., 13, 91 - 97 (2007).

29. A. Ankiewicz and N. Akhmediev, Comparison of Lagrangian approach and method of moments for reducing dimensionality of soliton dynamical systems, Chaos, 18, 033129 (2008).

30. W. Chang, J. M. Soto-Crespo, A. Ankiewicz and N. Akhmediev, Dissipative soliton resonances in anomalous dispersion regime, Phys. Rev. A, 77, 033840 (2009).

31. G. P. Agrawal Nonlinear Fiber Optics, 2nd edn, Academic Press Inc., San Diego, CA (1995).

32. Adrian Ankiewicz, J. M. Soto-Crespo, M. A. Chowdhury and N. Akhmediev, Rogue waves in optical fibers in presence of third order dispersion, self-steepening and self-frequency shift , J. Opt. Soc. Am. B, 30, 87-94 (2013).

33. V. V. Voronovich, V. I. Shrira and G. Thomas, Can bottom friction suppress 'freak wave' formation?, J. Fluid Mech. (2008), 604, 263 296. (2008).

34. A. Ankiewicz, D. J. Kedziora, A. Chowdury, U. Bandelow and N. Akhmediev, Infinite hierarchy of nonlinear Schrödinger equations and their solutions, Phys. Rev. E, 93, 012206 (2016).

35. P. M. Morse and H. Feshbach, Methods of theoretical physics, pp. 276 and 314, (McGraw-Hill book co., 1953).

36. A. D. Boardman and L. Velasco, Gyroelectric cubic-quintic dissipative solitons, IEEE J. Selected Topics in Quantum Electronics, 12, 388, (2006).

37. N. Akhmediev and A. Ankiewicz, Dissipative Solitons in the Complex Ginzburg-Landau and Swift-Hohenberg Equations, In: Dissipative Solitons, (Springer, Heidelberg, 2005).

38. A. Ankiewicz, J. M. Soto-Crespo, and N. Akhmediev, Rogue waves and rational solutions of the Hirota equation, Phys. Rev. E 81, 046602 (2010).

39. A. Ankiewicz and N. Akhmediev, Rogue wave solutions for the infinite integrable nonlinear Schrödinger equation hierarchy, Phys. Rev. E, 96, 012219 (2017).

40. N. Akhmediev and A. Ankiewicz, Solitons: Nonlinear Pulses and Beams, (Chap. \& Hall, London, 1997).

41. R. Hirota, Exact envelope-soliton solutions of a nonlinear wave equation, J. Math. Phys. 14, 805 (1973).

42. M. Lakshmanan, K. Porsezian, M. Daniel, Effect of Discreteness on the Continuum Limit of the Heisenberg Spin Chain, Phys. Lett. A, 133, $483-488$ (1988).

43. A. Ankiewicz, Yan Wang, S. Wabnitz and N. Akhmediev, Phys. Rev., E 89, 012907, (2014).

44. A. Ankiewicz, Int. J. Nonlinear Opt. Phys and Materials, 4, 857 - 870, (1995).

45. L. Gagnon and P. A. Belanger, Soliton self-frequency shift versus Galilean-like symmetry, Opt. Lett. 15, 466 - 468 (1990), see Eq.(13).

46. J. P. Gordon, Theory of the soliton self-frequency shift, Opt. Lett., 11, 662, (1986).

47. N. Akhmediev, W. Krolikowski, A. J. Lowery, Influence of the Ramaneffect on solitons in optical fibers, Opt. Commun., 131, $260-266$ (1996).

48. M. Facão, M. I. Carvalho, D. F. Parker, Soliton self-frequency shift: Selfsimilar solutions and their stability, Phys. Rev. E 81, 046604 (2010).

49. B. Jalali, D. Solli, K. Goda, K. Tsia \& C. Ropers, Real-time measurements, rare events and photon economics. Europ. Phys. J. - Special Topics, 185, 145 (2010).

50. A. Bhushan, F. Coppinger \& B. Jalali, Time-stretched analogue-todigital conversion, Electron. Lett., 34, 1081 (1998).

51. F. Coppinger, A. Bhushan \& B. Jalali, Photonic time stretch and its application to analog-to-digital conversion, IEEE Trans. Microwave Theory and Techniques, 47, 1309 (1999).

52. P. Suret, R. El Koussaifi, A. Tikan, C. Evain, S. Randoux, C. Szwaj \& S. Bielawski, Single-shot observation of optical rogue waves in integrable turbulence using time microscopy, Nature Commun., 7, 13136 (2016). 
53. M. Närhi, B. Wetzel, C. Billet, S. Toenger, T. Sylvestre, J.-M. Merolla, R. Morandotti, F. Dias, G. Genty \& J. M. Dudley, Real-time measurements of spontaneous breathers and rogue wave events in optical fibre modulation instability, Nature Commun., 7, 13675 (2016). 